\title{
A New Approximation Scheme Combining the Viscosity Method with Extragradient Method for Mixed Equilibrium Problems
}

\author{
Jian-Wen Peng ${ }^{1}$ and Soon-Yi Wu ${ }^{2}$ \\ ${ }^{1}$ College of Mathematics and Computer Science, Chongqing Normal University, Chongqing 400047, China \\ 2 Department of Mathematics, National Cheng Kung University, Tainan 701, Taiwan
}

Correspondence should be addressed to Jian-Wen Peng, jwpeng6@yahoo.com.cn

Received 8 November 2009; Revised 2 December 2009; Accepted 6 December 2009

Recommended by Simeon Reich

\begin{abstract}
We introduce a new approximation scheme combining the viscosity method with extragradient method for finding a common element of the set of solutions of a mixed equilibrium problem and the set of fixed points of a finite family of nonexpansive mappings and the set of the variational inequality for a monotone, Lipschitz continuous mapping. We obtain a strong convergence theorem for the sequences generated by these processes in Hilbert spaces. Based on this result, we also get some new and interesting results. The results in this paper generalize, extend, and improve some well-known results in the literature.
\end{abstract}

Copyright $\odot 2009$ J.-W. Peng and S.-Y. Wu. This is an open access article distributed under the Creative Commons Attribution License, which permits unrestricted use, distribution, and reproduction in any medium, provided the original work is properly cited.

\section{Introduction}

Let $H$ be a real Hilbert space with inner product $\langle\cdot, \cdot\rangle$ and induced norm $\|\cdot\|$ and let $C$ be a nonempty closed convex subset of $H$. Let $\varphi: C \rightarrow R \cup\{+\infty\}$ be a function and let $F$ be a bifunction from $C \times C$ to $R$, where $R$ is the set of real numbers. Ceng and Yao [1] and Bigi et al. [2] considered the following mixed equilibrium problem:

$$
\text { Find } x \in C \text { such that } F(x, y)+\varphi(y) \geq \varphi(x), \quad \forall y \in C \text {. }
$$

The set of solutions of (1.1) is denoted by $\operatorname{MEP}(F, \varphi)$. It is easy to see that $x$ is a solution of problem (1.1) implies that $x \in \operatorname{dom} \varphi=\{x \in C \mid \varphi(x)<+\infty\}$. 

problem:

If $\varphi=0$, then the mixed equilibrium problem (1.1) becomes the following equilibrium

Finding $x \in C$ such that $F(x, y) \geq 0, \quad \forall y \in C$.

The set of solutions of (1.2) is denoted by $E P(F)$.

If $F(x, y)=0$ for all $x, y \in C$, the mixed equilibrium problem (1.1) becomes the following minimization problem:

Finding $x \in C \quad$ such that $\varphi(y) \geq \varphi(x), \quad \forall y \in C$.

The set of solutions of (1.3) is denoted by $\operatorname{Argmin}(\varphi)$.

The problem (1.1) is very general in the sense that it includes, as special cases, optimization problems, variational inequalities, minimax problems, Nash equilibrium problem in noncooperative games, and others; see, for instance, [1-4].

Recall that a mapping $S$ of a closed convex subset $C$ into itself is nonexpansive [5] if there holds that

$$
\|S x-S y\| \leq\|x-y\| \quad \forall x, y \in C .
$$

We denote the set of fixed points of $S$ by Fix(S). Ceng and Yao [1] introduced an iterative scheme for finding a common element of the set of solutions of problem (1.1) and the set of common fixed points of a finite family of nonexpansive mappings in a Hilbert space and obtained a strong convergence theorem.

Some methods have been proposed to solve the problem (1.2); see, for instance, [3, 4, 6-12] and the references therein. Recently, Combettes and Hirstoaga [6] introduced an iterative scheme of finding the best approximation to the initial data when $\mathrm{EP}(F)$ is nonempty and proved a strong convergence theorem. Takahashi and Takahashi [7] introduced an iterative scheme by the viscosity approximation method for finding a common element of the set of solutions of problem (1.2) and the set of fixed points of a nonexpansive mapping in a Hilbert space and proved a strong convergence theorem. Su et al. [8] introduced the following iterative scheme by the viscosity approximation method for finding a common element of the set of solutions of problem (1.2) and the set of fixed points of a nonexpansive mapping and the set of solutions of the variational inequality problem for an $\alpha$-inverse strongly monotone mapping in a Hilbert space. Starting with an arbitrary $x_{1} \in H$, define sequences $\left\{x_{n}\right\}$ and $\left\{u_{n}\right\}$ by

$$
\begin{gathered}
F\left(u_{n}, y\right)+\frac{1}{r_{n}}\left\langle y-u_{n}, u_{n}-x_{n}\right\rangle \geq 0, \quad \forall y \in C, \\
x_{n+1}=\alpha_{n} f\left(x_{n}\right)+\left(1-\alpha_{n}\right) S P_{C}\left(u_{n}-\lambda_{n} A u_{n}\right), \quad \forall n \in N .
\end{gathered}
$$

They proved that under certain appropriate conditions imposed on $\left\{\alpha_{n}\right\},\left\{r_{n}\right\}$, and $\left\{\lambda_{n}\right\}$, the sequences $\left\{x_{n}\right\}$ and $\left\{u_{n}\right\}$ generated by (1.5) converge strongly to $z \in \operatorname{Fix}(S) \cap \operatorname{EP}(F) \cap \operatorname{VI}(C, A)$, 
where $z=P_{\mathrm{Fix}(S) \cap \mathrm{EP}(F) \cap \mathrm{VI}(C, A)} f(z)$. Tada and Takahashi [9] introduced two iterative schemes for finding a common element of the set of solutions of problem (1.2) and the set of fixed points of a nonexpansive mapping in a Hilbert space and obtained both strong convergence theorem and weak convergence theorem.

On the other hand, for solving the variational inequality problem in the finitedimensional Euclidean $R^{n}$, Korpelevich [13] introduced the following so-called extragradient method:

$$
\begin{gathered}
x_{1}=x \in C, \\
y_{n}=P_{C}\left(x_{n}-\lambda A x_{n}\right), \\
x_{n+1}=P_{C}\left(x_{n}-\lambda A y_{n}\right)
\end{gathered}
$$

for every $n=0,1,2, \ldots$, where $\lambda \in(0,1 / k)$. She showed that if $\operatorname{VI}(C, A)$ is nonempty, then the sequences $\left\{x_{n}\right\}$ and $\left\{y_{n}\right\}$, generated by (1.6), converge to the same point $z \in \operatorname{VI}(C, A)$. The idea of the extragradient iterative process introduced by Korpelevich was successfully generalized and extended not only in Euclidean but also in Hilbert and Banach spaces; see, for example, the recent papers of He et al. [14], Gárciga Otero and Iuzem [15], and Solodov and Svaiter [16], Solodov [17]. Moreover, Zeng and Yao [18] and Nadezhkina and Takahashi [19] introduced iterative processes based on the extragradient method for finding the common element of the set of fixed points of nonexpansive mappings and the set of solutions of variational inequality problem for a monotone, Lipschitz continuous mapping. Yao and Yao [20] introduced an iterative process based on the extragradient method for finding the common element of the set of fixed points of nonexpansive mappings and the set of solutions of variational inequality problem for an $\alpha$-inverse strongly monotone mapping. Plubtieng and Punpaeng [11] introduced an iterative process based on the extragradient method for finding the common element of the set of fixed points of nonexpansive mappings, the set of solutions of an equilibrium problem, and the set of solutions of variational inequality problem for $\alpha$-inverse strongly monotone mappings. Chang et al. [12] introduced some iterative processes based on the extragradient method for finding the common element of the set of fixed points of a infinite family of nonexpansive mappings, the set of solutions of an equilibrium problem, and the set of solutions of variational inequality problem for an $\alpha$-inverse strongly monotone mapping. Peng et al. [21] introduced a new approximation scheme combining the viscosity method with parallel method for finding a common element of the set of solutions of a generalized equilibrium problem and the set of fixed points of a finite family of strict pseudocontractions and obtain a strong convergence theorem for the sequences generated by these processes in Hilbert spaces.

In the present paper, we introduce a new approximation scheme combining the viscosity method with extragradient method for finding a common element of the set of solutions of a mixed equilibrium problem, the set of fixed points of a finite family of nonexpansive mappings, and the set of solutions of the variational inequality for a monotone, Lipschitz continuous mapping. We obtain a strong convergence theorem for the sequences generated by these processes. Based on this result, we also get some new and interesting results. The results in this paper generalize and improve some well-known results in the literature. 


\section{Preliminaries}

Let $H$ be a real Hilbert space with inner product $\langle\cdot, \cdot\rangle$ and norm $\|\cdot\|$. Let $C$ be a nonempty closed convex subset of $H$. Let symbols $\rightarrow$ and $\rightarrow$ denote strong and weak convergence, respectively. In a real Hilbert space $H$, it is well known that

$$
\|\lambda x+(1-\lambda) y\|^{2}=\lambda\|x\|^{2}+(1-\lambda)\|y\|^{2}-\lambda(1-\lambda)\|x-y\|^{2}
$$

for all $x, y \in H$ and $\lambda \in[0,1]$.

For any $x \in H$, there exists a unique nearest point in $C$, denoted by $P_{C}(x)$, such that $\left\|x-P_{C}(x)\right\| \leq\|x-y\|$ for all $y \in C$. The mapping $P_{C}$ is called the metric projection of $H$ onto $C$. We know that $P_{C}$ is a nonexpansive mapping from $H$ onto $C$. It is also known that $P_{C} x \in C$ and

$$
\left\langle x-P_{C}(x), P_{C}(x)-y\right\rangle \geq 0
$$

for all $x \in H$ and $y \in C$.

It is easy to see that (2.2) is equivalent to

$$
\|x-y\|^{2} \geq\left\|x-P_{C}(x)\right\|^{2}+\left\|y-P_{C}(x)\right\|^{2}
$$

for all $x \in H$ and $y \in C$.

A mapping $A$ of $C$ into $H$ is called monotone if

$$
\langle A x-A y, x-y\rangle \geq 0
$$

for all $x, y \in C$. A mapping $A$ of $C$ into $H$ is called $\alpha$-inverse strongly monotone if there exists a positive real number $\alpha$ such that

$$
\langle x-y, A x-A y\rangle \geq \alpha\|A x-A y\|^{2}
$$

for all $x, y \in C$. A mapping $A: C \rightarrow H$ is called $k$-Lipschitz continuous if there exists a positive real number $k$ such that

$$
\|A x-A y\| \leq k\|x-y\|
$$

for all $x, y \in C$. It is easy to see that if $A$ is $\alpha$-inverse strongly monotone mappings, then $A$ is monotone and Lipschitz continuous. The converse is not true in general. The class of $\alpha$ inverse strongly monotone mappings does not contain some important classes of mappings even in a finite-dimensional case. For example, if the matrix in the corresponding linear complementarity problem is positively semidefinite, but not positively definite, then the mapping $A$ is monotone and Lipschitz continuous, but not $\alpha$-inverse strongly monotone. 
Let $A$ be a monotone mapping of $C$ into $H$. In the context of the variational inequality problem the characterization of projection (2.2) implies the following:

$$
\begin{gathered}
u \in \mathrm{VI}(C, A) \Longrightarrow u=P_{C}(u-\lambda A u), \quad \lambda>0, \\
u=P_{C}(u-\lambda A u) \quad \text { for some } \lambda>0 \Longrightarrow u \in \operatorname{VI}(C, A) .
\end{gathered}
$$

It is also known that $H$ satisfies Opial's condition [22], that is, for any sequence $\left\{x_{n}\right\} \subset$ $H$ with $x_{n} \rightarrow x$, the inequality

$$
\liminf _{n \rightarrow \infty}\left\|x_{n}-x\right\|<\liminf _{n \rightarrow \infty}\left\|x_{n}-y\right\|
$$

holds for every $y \in H$ with $x \neq y$.

A set-valued mapping $T: H \rightarrow 2^{H}$ is called monotone if for all $x, y \in H, f \in T x$ and $g \in T y$ imply $\langle x-y, f-g\rangle \geq 0$. A monotone mapping $T: H \rightarrow 2^{H}$ is maximal if its graph $G(T)$ of $T$ is not properly contained in the graph of any other monotone mapping. It is known that a monotone mapping $T$ is maximal if and only if for $(x, f) \in H \times H,\langle x-y, f-g\rangle \geq 0$ for every $(y, g) \in G(T)$ implies $f \in T x$. Let A be a monotone, $k$-Lipschitz continuous mapping of $C$ into $H$ and let $N_{C} v$ be normal cone to $C$ at $v \in C$, that is, $N_{C} v=\{w \in H:\langle v-u, w\rangle \geq 0, \forall u \in C\}$. Define

$$
T v= \begin{cases}A v+N_{C} v & \text { if } v \in C, \\ \emptyset & \text { if } v \notin C .\end{cases}
$$

Then $T$ is maximal monotone and $0 \in T v$ if and only if $v \in \operatorname{VI}(C, A)$ (see [23]).

For solving the mixed equilibrium problem, let us give the following assumptions for the bifunction $F, \varphi$ and the set $C$ :

(A1) $F(x, x)=0$ for all $x \in C$;

(A2) $F$ is monotone, that is, $F(x, y)+F(y, x) \leq 0$ for any $x, y \in C$;

(A3) for each $y \in C, x \mapsto F(x, y)$ is weakly upper semicontinuous;

(A4) for each $x \in C, y \mapsto F(x, y)$ is convex;

(A5) for each $x \in C, y \mapsto F(x, y)$ is lower semicontinuous;

(B1) for each $x \in H$ and $r>0$, there exist a bounded subset $D_{x} \subseteq C$ and $y_{x} \in C$ such that for any $z \in C \backslash D_{x}$,

$$
F\left(z, y_{x}\right)+\varphi\left(y_{x}\right)+\frac{1}{r}\left\langle y_{x}-z, z-x\right\rangle<\varphi(z)
$$

(B2) $C$ is a bounded set;

(B3) for each $x \in H$ and $r>0$, there exist a bounded subset $D_{x} \subseteq C$ and $y_{x} \in C$ such that for any $z \in C \backslash D_{x}$,

$$
\varphi\left(y_{x}\right)+\frac{1}{r}\left\langle y_{x}-z, z-x\right\rangle<\varphi(z)
$$


(B4) for each $x \in H$ and $r>0$, there exist a bounded subset $D_{x} \subseteq C$ and $y_{x} \in C$ such that for any $z \in C \backslash D_{x}$,

$$
F\left(z, y_{x}\right)+\frac{1}{r}\left\langle y_{x}-z, z-x\right\rangle<0
$$

We will use the following results in the sequel.

Lemma 2.1 (see $[21,24]$ ). Let $C$ be a nonempty closed convex subset of $H$. Let $F$ be a bifunction from $C \times C$ to $R$ satisfying (A1)-(A5) and let $\varphi: C \rightarrow R \cup\{+\infty\}$ be a proper lower semicontinuous and convex function. Assume that either (B1) or (B2) holds. For $r>0$ and $x \in H$, define a mapping $T_{r}: H \rightarrow C$ as follows:

$$
T_{r}(x)=\left\{z \in C: F(z, y)+\varphi(y)+\frac{1}{r}\langle y-z, z-x\rangle \geq \varphi(z), \forall y \in C\right\}
$$

for all $x \in H$. Then the following conclusions hold:

(1) for each $x \in H, T_{r}(x) \neq \emptyset$;

(2) $T_{r}$ is single-valued;

(3) $T_{r}$ is firmly nonexpansive, that is, for any $x, y \in H$,

$$
\left\|T_{r}(x)-T_{r}(y)\right\|^{2} \leq\left\langle T_{r}(x)-T_{r}(y), x-y\right\rangle ;
$$

(4) $\operatorname{Fix}\left(T_{r}\right)=\operatorname{MEP}(F, \varphi)$;

(5) $\operatorname{MEP}(F, \varphi)$ is closed and convex.

Lemma 2.2 (see $[25,26]$ ). Assume that $\left\{\alpha_{n}\right\}$ is a sequence of nonnegative real numbers such that

$$
\alpha_{n+1} \leq\left(1-\gamma_{n}\right) \alpha_{n}+\delta_{n}
$$

where $\gamma_{n}$ is a sequence in $(0,1)$ and $\left\{\delta_{n}\right\}$ is a sequence such that

(i) $\sum_{n=1}^{\infty} \gamma_{n}=\infty$;

(ii) $\lim \sup _{n \rightarrow \infty} \delta_{n} / \gamma_{n} \leq 0$ or $\sum_{n=1}^{\infty}\left|\delta_{n}\right|<\infty$.

Then, $\lim _{n \rightarrow \infty} \alpha_{n}=0$.

Lemma 2.3. In a real Hilbert space $H$, there holds the following inequality:

$$
\|x+y\|^{2} \leq\|x\|^{2}+2\langle y, x+y\rangle
$$

for all $x, y \in H$. 
Lemma 2.4 (see [21]). Let $\left\{x_{n}\right\}$ and $\left\{w_{n}\right\}$ be bounded sequences in a Banach space, and let $\left\{\beta_{n}\right\}$ be a sequence of real numbers such that $0<\liminf _{n \rightarrow \infty} \beta_{n} \leq \limsup _{n \rightarrow \infty} \beta_{n}<1$ for all $n=0,1,2, \ldots$. Suppose that $x_{n+1}=\left(1-\beta_{n}\right) w_{n}+\beta_{n} x_{n}$ for all $n=0,1,2, \ldots$ and $\lim \sup _{n \rightarrow \infty}\left\|w_{n+1}-w_{n}\right\|+\| x_{n+1}-$ $x_{n} \| \leq 0$. Then, $\lim _{n \rightarrow \infty}\left\|w_{n}-x_{n}\right\|=0$.

Let $\left\{T_{i}\right\}_{i=1}^{N}$ be a finite family of nonexpansive mappings of $C$ into itself and let $\lambda_{1}, \lambda_{2}, \ldots, \lambda_{N}$ be real numbers such that $0 \leq \lambda_{i} \leq 1$ for every $i=1,2, \ldots, N$. We define a mapping $W$ of $C$ into itself as follows:

$$
\begin{gathered}
U_{1}=\lambda_{1} T_{1}+\left(1-\lambda_{1}\right) I, \\
U_{2}=\lambda_{2} T_{2} U_{1}+\left(1-\lambda_{2}\right) I, \\
\vdots \\
U_{N-1}=\lambda_{N-1} T_{N-1} U_{N-2}+\left(1-\lambda_{N-1}\right) I, \\
W:=U_{N}=\lambda_{N} T_{N} U_{N-1}+\left(1-\lambda_{N}\right) I .
\end{gathered}
$$

Such a mapping $W$ is called the $W$-mapping generated by $T_{1}, T_{2}, \ldots, T_{N}$ and $\lambda_{1}, \lambda_{2}, \ldots, \lambda_{N}$. It is easy to see that nonexpansivity of each $T_{i}$ ensures the nonexpansivity of $W$. The concept of $W$-mappings was introduced in [27, 28]. It is now one of the main tools in studying convergence of iterative methods for approaching a common fixed point of nonlinear mappings; more recent progresses can be found in $[10,29,30]$ and the references cited therein.

Lemma 2.5 (see [29]). Let $C$ be a nonempty closed convex set of a strictly convex Banach space. Let $T_{1}, T_{2}, \ldots, T_{N}$ be nonexpansive mappings of $C$ into itself such that $\bigcap_{i=1}^{N} F\left(T_{i}\right) \neq \emptyset$ and let $\lambda_{1}, \lambda_{2}, \ldots, \lambda_{N}$ be real numbers such that $0<\lambda_{i}<1$ for every $i=1,2, \ldots, N-1$ and $0<\lambda_{N} \leq 1$. Let $W$ be the $W$-mapping generated by $T_{1}, T_{2}, \ldots, T_{N}$ and $\lambda_{1}, \lambda_{2}, \ldots, \lambda_{N}$. Then $\operatorname{Fix}(W)=\bigcap_{i=1}^{N} \operatorname{Fix}\left(T_{i}\right)$.

Lemma 2.6 (see [10]). Let $C$ be a nonempty convex subset of a Banach space. Let $\left\{T_{i}\right\}_{i=1}^{N}$ be a finite family of nonexpansive mappings of $C$ into itself and let $\left\{\lambda_{n, 1}\right\}\left\{\lambda_{n, 2}\right\}, \ldots,\left\{\lambda_{n, N}\right\}$ be sequences in $[0,1]$ such that $\lambda_{n, i} \rightarrow \lambda_{i}(i=1, \ldots, N)$. Moreover for every integer $n \geq 1$, let $W$ and $W_{n}$ be the $W$-mappings generated by $T_{1}, T_{2}, \ldots, T_{N}$ and $\lambda_{1}, \lambda_{2}, \ldots, \lambda_{N}$ and $T_{1}, T_{2}, \ldots, T_{N}$ and $\left\{\lambda_{n, 1}\right\}\left\{\lambda_{n, 2}\right\}, \ldots,\left\{\lambda_{n, N}\right\}$, respectively. Then for every $x \in C$, it follows that

$$
\lim _{n \rightarrow \infty}\left\|W_{n} x-W x\right\|=0
$$

\section{Strong Convergence Theorems}

In this section, we show a strong convergence of an iterative algorithm based on both viscosity approximation method and extragradient method which solves the problem of finding a common element of the set of solutions of a mixed equilibrium problem, the set of fixed points of a finite family of nonexpansive mappings, and the set of solutions of the variational inequality for a monotone, Lipschitz continuous mapping in a Hilbert space. 
Theorem 3.1. Let $C$ be a nonempty closed convex subset of a real Hilbert space $H$. Let $F$ be a bifunction from $C \times C$ to $R$ satisfying (A1)-(A5) and let $\varphi: C \rightarrow R \cup\{+\infty\}$ be a proper lower semicontinuous and convex function. Let $A$ be a monotone and $k$-Lipschitz continuous mapping of $C$ into $H$. Let $T_{1}, T_{2}, \ldots, T_{N}$ be a finite family of nonexpansive mappings of $C$ into $H$ such that $\Omega=\bigcap_{n=1}^{N} \operatorname{Fix}\left(T_{i}\right) \cap \operatorname{VI}(C, A) \cap \operatorname{MEP}(F, \varphi) \neq \emptyset$. Let $\left\{\lambda_{n, 1}\right\},\left\{\lambda_{n, 2}\right\}, \ldots,\left\{\lambda_{n, N}\right\}$ be sequences in $\left[\varepsilon_{1}, \varepsilon_{2}\right]$ with $0<\varepsilon_{1} \leq \varepsilon_{2}<1$. Let $W_{n}$ be the $W$-mapping generated by $T_{1}, T_{2}, \ldots, T_{N}$ and $\lambda_{n, 1}, \lambda_{n, 2}, \ldots, \lambda_{n, N}$. Assume that either (B1) or (B2) holds. Let $f$ be a contraction of $H$ into itself and let $\left\{x_{n}\right\},\left\{u_{n}\right\}$, and $\left\{y_{n}\right\}$ be sequences generated by

$$
\begin{gathered}
x_{1}=x \in C, \\
F\left(u_{n}, y\right)+\varphi(y)-\varphi\left(u_{n}\right)+\frac{1}{r_{n}}\left\langle y-u_{n}, u_{n}-x_{n}\right\rangle \geq 0, \quad \forall y \in C \\
y_{n}=P_{C}\left(u_{n}-\gamma_{n} A u_{n}\right), \\
x_{n+1}=\alpha_{n} f\left(x_{n}\right)+\beta_{n} x_{n}+\left(1-\alpha_{n}-\beta_{n}\right) W_{n} P_{C}\left(u_{n}-\gamma_{n} A y_{n}\right)
\end{gathered}
$$

for every $n=1,2, \ldots$ where $\left\{\gamma_{n}\right\},\left\{r_{n}\right\},\left\{\alpha_{n}\right\},\left\{\lambda_{n 1}\right\},\left\{\lambda_{n 2}\right\}, \ldots,\left\{\lambda_{n N}\right\}$, and $\left\{\beta_{n}\right\}$ are sequences of numbers satisfying the conditions:

(C1) $\lim _{n \rightarrow \infty} \alpha_{n}=0$ and $\sum_{n=1}^{\infty} \alpha_{n}=\infty$;

(C2) $1>\lim \sup _{n \rightarrow \infty} \beta_{n} \geq \liminf _{n \rightarrow \infty} \beta_{n}>0$;

(C3) $\lim _{n \rightarrow \infty} \gamma_{n}=0$;

(C4) $\liminf _{n \rightarrow \infty} r_{n}>0$ and $\lim _{n \rightarrow \infty}\left|r_{n+1}-r_{n}\right|=0$;

(C5) $\lim _{n \rightarrow \infty}\left|\lambda_{n, i}-\lambda_{n-1, i}\right|=0$ for all $i=1,2, \ldots, N$.

Then, $\left\{x_{n}\right\},\left\{u_{n}\right\}$, and $\left\{y_{n}\right\}$ converge strongly to $w=P_{\Omega} f(w)$.

Proof. We show that $P_{\Omega} f$ is a contraction of $C$ into itself. In fact, there exists $a \in[0,1)$ such that $\|f(x)-f(y)\| \leq a\|x-y\|$ for all $x, y \in C$. So, we have

$$
\left\|P_{\Omega} f(x)-P_{\Omega} f(y)\right\| \leq\|f(x)-f(y)\| \leq a\|x-y\|
$$

for all $x, y \in C$. Since $H$ is complete, there exists a unique element $u_{0} \in C$ such that $u_{0}=$ $P_{\Omega} f\left(u_{0}\right)$. 
Put $t_{n}=P_{C}\left(u_{n}-\gamma_{n} A y_{n}\right)$ for every $n=1,2, \ldots$ Let $u \in \Omega$ and let $\left\{T_{r_{n}}\right\}$ be a sequence of mappings defined as in Lemma 2.1. Then $u=P_{C}\left(u-\gamma_{n} A u\right)$. From $u_{n}=T_{r_{n}}\left(x_{n}\right) \in C$, we have

$$
\left\|u_{n}-u\right\|=\left\|T_{r_{n}}\left(x_{n}\right)-T_{r_{n}}(u)\right\| \leq\left\|x_{n}-u\right\|
$$

From (2.3), the monotonicity of $A$, and $u \in \operatorname{VI}(C, A)$, we have

$$
\begin{aligned}
\| t_{n}- & u \|^{2} \\
& \leq\left\|u_{n}-\gamma_{n} A y_{n}-u\right\|^{2}-\left\|u_{n}-\gamma_{n} A y_{n}-t_{n}\right\|^{2} \\
& =\left\|u_{n}-u\right\|^{2}-\left\|u_{n}-t_{n}\right\|^{2}+2 \gamma_{n}\left\langle A y_{n}, u-t_{n}\right\rangle \\
& =\left\|u_{n}-u\right\|^{2}-\left\|u_{n}-t_{n}\right\|^{2}+2 \gamma_{n}\left(\left\langle A y_{n}-A u, u-y_{n}\right\rangle+\left\langle A u, u-y_{n}\right\rangle+\left\langle A y_{n}, y_{n}-t_{n}\right\rangle\right) \\
& \leq\left\|u_{n}-u\right\|^{2}-\left\|u_{n}-t_{n}\right\|^{2}+2 \gamma_{n}\left\langle A y_{n}, y_{n}-t_{n}\right\rangle \\
& \leq\left\|u_{n}-u\right\|^{2}-\left\|u_{n}-y_{n}\right\|^{2}-2\left\langle u_{n}-y_{n}, y_{n}-t_{n}\right\rangle-\left\|y_{n}-t_{n}\right\|^{2}+2 \gamma_{n}\left\langle A y_{n}, y_{n}-t_{n}\right\rangle \\
& =\left\|u_{n}-u\right\|^{2}-\left\|u_{n}-y_{n}\right\|^{2}-\left\|y_{n}-t_{n}\right\|^{2}+2\left\langle u_{n}-\gamma_{n} A y_{n}-y_{n}, t_{n}-y_{n}\right\rangle .
\end{aligned}
$$

Further, Since $y_{n}=P_{C}\left(u_{n}-\gamma_{n} A u_{n}\right)$ and $A$ is $k$-Lipschitz continuous, we have

$$
\begin{aligned}
\left\langle u_{n}-\gamma_{n} A y_{n}-y_{n}, t_{n}-y_{n}\right\rangle & =\left\langle u_{n}-\gamma_{n} A u_{n}-y_{n}, t_{n}-y_{n}\right\rangle+\left\langle\gamma_{n} A u_{n}-\gamma_{n} A y_{n}, t_{n}-y_{n}\right\rangle \\
& \leq\left\langle\gamma_{n} A u_{n}-\gamma_{n} A y_{n}, t_{n}-y_{n}\right\rangle \leq \gamma_{n} k\left\|u_{n}-y_{n}\right\|\left\|t_{n}-y_{n}\right\| .
\end{aligned}
$$

So, it follows from (C3) that the following inequality holds for $n \geq n_{0}$, where $n_{0}$ is a positive integer:

$$
\begin{aligned}
\left\|t_{n}-u\right\|^{2} & \leq\left\|u_{n}-u\right\|^{2}-\left\|u_{n}-y_{n}\right\|^{2}-\left\|y_{n}-t_{n}\right\|^{2}+2 \gamma_{n} k\left\|u_{n}-y_{n}\right\|\left\|t_{n}-y_{n}\right\| \\
& \leq\left\|u_{n}-u\right\|^{2}-\left\|u_{n}-y_{n}\right\|^{2}-\left\|y_{n}-t_{n}\right\|^{2}+\gamma_{n}{ }^{2} k^{2}\left\|u_{n}-y_{n}\right\|^{2}+\left\|t_{n}-y_{n}\right\|^{2} \\
& =\left\|u_{n}-u\right\|^{2}+\left(\gamma_{n}{ }^{2} k^{2}-1\right)\left\|u_{n}-y_{n}\right\|^{2} \\
& \leq\left\|u_{n}-u\right\|^{2} .
\end{aligned}
$$

Put $M_{0}=\max \left\{\left\|x_{1}-u\right\|,(1 /(1-a))\|f(u)-u\|\right\}$. It is obvious that $\left\|x_{1}-u\right\| \leq M_{0}$. Suppose $\left\|x_{n}-u\right\| \leq M_{0}$. By Lemma 2.5, we know that $W_{n}$ is nonexpansive and $\operatorname{Fix}\left(W_{n}\right)=\bigcap_{i=1}^{N} \operatorname{Fix}\left(T_{i}\right)$. 
From (3.3), (3.6) and $x_{n+1}=\alpha_{n} f\left(x_{n}\right)+\beta_{n} x_{n}+\left(1-\alpha_{n}-\beta_{n}\right) W_{n} t_{n}$, we have $u=W_{n} u$ and

$$
\begin{aligned}
\left\|x_{n+1}-u\right\| & =\left\|\alpha_{n} f\left(x_{n}\right)+\beta_{n} x_{n}+\left(1-\alpha_{n}-\beta_{n}\right) W_{n} t_{n}-u\right\| \\
& \leq \alpha_{n}\left\|f\left(x_{n}\right)-f(u)\right\|+\alpha_{n}\|f(u)-u\|+\beta_{n}\left\|x_{n}-u\right\|+\left(1-\alpha_{n}-\beta_{n}\right)\left\|W_{n} t_{n}-u\right\| \\
& \leq \alpha_{n} a\left\|x_{n}-u\right\|+\alpha_{n}\|f(u)-u\|+\beta_{n}\left\|x_{n}-u\right\|+\left(1-\alpha_{n}-\beta_{n}\right)\left\|W_{n} t_{n}-u\right\| \\
& \leq \alpha_{n} a\left\|x_{n}-u\right\|+\alpha_{n}\|f(u)-u\|+\beta_{n}\left\|x_{n}-u\right\|+\left(1-\alpha_{n}-\beta_{n}\right)\left\|t_{n}-u\right\| \\
& \leq \alpha_{n} a\left\|x_{n}-u\right\|+\alpha_{n}\|f(u)-u\|+\beta_{n}\left\|x_{n}-u\right\|+\left(1-\alpha_{n}-\beta_{n}\right)\left\|x_{n}-u\right\| \\
& =(1-a) \alpha_{n} \frac{\|f(u)-u\|}{1-a}+\left(1-(1-a) \alpha_{n}\right)\left\|x_{n}-u\right\| \\
& \leq(1-a) \alpha_{n} M_{0}+\left(1-(1-a) \alpha_{n}\right) M_{0}=M_{0}
\end{aligned}
$$

for every $n=1,2, \ldots$. Therefore, $\left\{x_{n}\right\}$ is bounded. From (3.3) and (3.6), we also obtain that $\left\{t_{n}\right\}$ and $\left\{u_{n}\right\}$ are bounded.

From $y_{n}=P_{C}\left(u_{n}-\gamma_{n} A u_{n}\right)$ and the monotonicity and the Lipschitz continuity of $A$, we have

$$
\begin{aligned}
\left\|y_{n}-u\right\|^{2} & =\left\|P_{C}\left(u_{n}-\gamma_{n} A u_{n}\right)-P_{C}\left(u-\gamma_{n} A u\right)\right\|^{2} \\
& \leq\left\|u_{n}-\gamma_{n} A u_{n}-\left(u-\gamma_{n} A u\right)\right\|^{2} \\
& =\left\|u_{n}-u\right\|^{2}-2 \gamma_{n}\left\langle A u_{n}-A u, u_{n}-u\right\rangle+\gamma_{n}^{2}\left\|A u_{n}-A u\right\|^{2} \\
& \leq\left\|u_{n}-u\right\|^{2}+\gamma_{n}^{2} k^{2}\left\|u_{n}-u\right\|^{2} \\
& =\left(1+\gamma_{n}^{2} k^{2}\right)\left\|u_{n}-u\right\|^{2} .
\end{aligned}
$$

Hence, we obtain that $\left\{y_{n}\right\}$ is bounded. It follows from the Lipschitz continuity of $A$ that $\left\{A x_{n}\right\},\left\{A u_{n}\right\}$, and $\left\{A y_{n}\right\}$ are bounded. Since $f$ and $W_{n}$ are nonexpansive, we know that $\left\{f\left(x_{n}\right)\right\}$ and $\left\{W_{n} t_{n}\right\}$ are also bounded. From the definition of $t_{n}$, we get

$$
\begin{aligned}
\left\|t_{n+1}-t_{n}\right\| & =\left\|P_{C}\left(u_{n+1}-\gamma_{n+1} A y_{n+1}\right)-P_{C}\left(u_{n}-\gamma_{n} A y_{n}\right)\right\| \\
& \leq\left\|\left(u_{n+1}-\gamma_{n+1} A y_{n+1}\right)-\left(u_{n}-\gamma_{n} A y_{n}\right)\right\| \\
& \leq\left\|\left(u_{n+1}-\gamma_{n+1} A u_{n+1}\right)-\left(u_{n}-\gamma_{n+1} A u_{n}\right)+\gamma_{n+1}\left(A u_{n+1}-A y_{n+1}-A u_{n}\right)+\gamma_{n} A y_{n}\right\| \\
& \leq\left\|u_{n+1}-u_{n}\right\|+\gamma_{n+1}\left\|A u_{n+1}-A u_{n}\right\|+\gamma_{n+1}\left\|A u_{n+1}-A y_{n+1}-A u_{n}\right\|+\gamma_{n}\left\|A y_{n}\right\| \\
& \leq\left\|u_{n+1}-u_{n}\right\|+k \gamma_{n+1}\left\|u_{n+1}-u_{n}\right\|+\gamma_{n+1}\left\|A u_{n+1}-A y_{n+1}-A u_{n}\right\|+\gamma_{n}\left\|A y_{n}\right\| \\
& \leq\left\|u_{n+1}-u_{n}\right\|+\left(\gamma_{n+1}+\gamma_{n}\right) M_{1}
\end{aligned}
$$


where $M_{1}$ is an approximate constant such that

$$
M_{1} \geq \sup _{n \geq 1}\left\{k\left\|u_{n+1}-u_{n}\right\|+\left\|A u_{n+1}-A y_{n+1}-A u_{n}\right\|+\left\|A y_{n}\right\|\right\} .
$$

On the other hand, from $u_{n}=T_{r_{n}}\left(x_{n}\right)$ and $u_{n+1}=T_{r_{n+1}}\left(x_{n+1}\right)$, we have

$$
\begin{gathered}
F\left(u_{n}, y\right)+\varphi(y)-\varphi\left(u_{n}\right)+\frac{1}{r_{n}}\left\langle y-u_{n}, u_{n}-x_{n}\right\rangle \geq 0, \quad \forall y \in C, \\
F\left(u_{n+1}, y\right)+\varphi(y)-\varphi\left(u_{n+1}\right)+\frac{1}{r_{n+1}}\left\langle y-u_{n+1}, u_{n+1}-x_{n+1}\right\rangle \geq 0, \quad \forall y \in C .
\end{gathered}
$$

Putting $y=u_{n+1}$ in (3.11) and $y=u_{n}$ in (3.12), we have

$$
\begin{gathered}
F\left(u_{n}, u_{n+1}\right)+\varphi\left(u_{n+1}\right)-\varphi\left(u_{n}\right)+\frac{1}{r_{n}}\left\langle u_{n+1}-u_{n}, u_{n}-x_{n}\right\rangle \geq 0, \\
F\left(u_{n+1}, u_{n}\right)+\varphi\left(u_{n}\right)-\varphi\left(u_{n+1}\right)+\frac{1}{r_{n+1}}\left\langle u_{n}-u_{n+1}, u_{n+1}-x_{n+1}\right\rangle \geq 0 .
\end{gathered}
$$

So, from the monotonicity of $F$, we get

$$
\left\langle u_{n+1}-u_{n}, \frac{u_{n}-x_{n}}{r_{n}}-\frac{u_{n+1}-x_{n+1}}{r_{n+1}}\right\rangle \geq 0
$$

and hence

$$
\left\langle u_{n+1}-u_{n}, u_{n}-u_{n+1}+u_{n+1}-x_{n}-\frac{r_{n}}{r_{n+1}}\left(u_{n+1}-x_{n+1}\right)\right\rangle \geq 0 .
$$

Without loss of generality, let us assume that there exists a real number $b$ such that $r_{n}>b>0$ for all $n \in N$. Then,

$$
\begin{aligned}
\left\|u_{n+1}-u_{n}\right\|^{2} & \leq\left\langle u_{n+1}-u_{n}, x_{n+1}-x_{n}+\left(1-\frac{r_{n}}{r_{n+1}}\right)\left(u_{n+1}-x_{n+1}\right)\right\rangle \\
& \leq\left\|u_{n+1}-u_{n}\right\|\left\{\left\|x_{n+1}-x_{n}\right\|+\left|1-\frac{r_{n}}{r_{n+1}}\right|\left\|u_{n+1}-x_{n+1}\right\|\right\},
\end{aligned}
$$

and hence

$$
\begin{aligned}
\left\|u_{n+1}-u_{n}\right\| & \leq\left\|x_{n+1}-x_{n}\right\|+\frac{1}{r_{n+1}}\left|r_{n+1}-r_{n}\right|\left\|u_{n+1}-x_{n+1}\right\| \\
& \leq\left\|x_{n+1}-x_{n}\right\|+\frac{1}{b}\left|r_{n+1}-r_{n}\right| M_{2}
\end{aligned}
$$

where $M_{2}=\sup \left\{\left\|u_{n}-x_{n}\right\|: n \in N\right\}$. 
It follows from (3.9) and (3.7) that

$$
\left\|t_{n+1}-t_{n}\right\| \leq\left\|x_{n+1}-x_{n}\right\|+\frac{1}{b}\left|r_{n+1}-r_{n}\right| M_{2}+\left(\gamma_{n+1}+\gamma_{n}\right) M_{1}
$$

Define a sequence $\left\{v_{n}\right\}$ such that

$$
x_{n+1}=\beta_{n} x_{n}+\left(1-\beta_{n}\right) v_{n}, \quad \forall n \geq 1 .
$$

Then, we have

$$
\begin{aligned}
v_{n+1}-v_{n}= & \frac{x_{n+2}-\beta_{n+1} x_{n+1}}{1-\beta_{n+1}}-\frac{x_{n+1}-\beta_{n} x_{n}}{1-\beta_{n}} \\
= & \frac{\alpha_{n+1} f\left(x_{n+1}\right)+\left(1-\alpha_{n+1}-\beta_{n+1}\right) W_{n+1} t_{n+1}}{1-\beta_{n+1}}-\frac{\alpha_{n} f\left(x_{n}\right)+\left(1-\alpha_{n}-\beta_{n}\right) W_{n} t_{n}}{1-\beta_{n}} \\
= & \frac{\alpha_{n+1}}{1-\beta_{n+1}} f\left(x_{n+1}\right)-\frac{\alpha_{n}}{1-\beta_{n}} f\left(x_{n}\right)+W_{n+1} t_{n+1}-W_{n} t_{n} \\
& +\frac{\alpha_{n}}{1-\beta_{n}} W_{n} t_{n}-\frac{\alpha_{n+1}}{1-\beta_{n+1}} W_{n+1} t_{n+1}
\end{aligned}
$$

Next we estimate $\left\|W_{n+1} t_{n+1}-W_{n} t_{n}\right\|$. It follows from the definition of $W_{n}$ that

$$
\begin{aligned}
\left\|W_{n+1} t_{n}-W_{n} t_{n}\right\|= & \left\|\lambda_{n+1, N} T_{N} U_{n+1, N-1} t_{n}+\left(1-\lambda_{n+1, N}\right) t_{n}-\lambda_{n, N} T_{N} U_{n, N-1} t_{n}-\left(1-\lambda_{n, N}\right) t_{n}\right\| \\
\leq & \left|\lambda_{n+1, N}-\lambda_{n, N}\right||| t_{n}\|+\| \lambda_{n+1, N} T_{N} U_{n+1, N-1} t_{n}-\lambda_{n, N} T_{N} U_{n, N-1} t_{n} \| \\
\leq & \left|\lambda_{n+1, N}-\lambda_{n, N}\right|\left\|t_{n}\right\|+\left\|\lambda_{n+1, N}\left(T_{N} U_{n+1, N-1} t_{n}-T_{N} U_{n, N-1} t_{n}\right)\right\| \\
& +\left|\lambda_{n+1, N}-\lambda_{n, N}\right|\left\|T_{N} U_{n, N-1} t_{n}\right\| \\
\leq & 2 M_{3}\left|\lambda_{n+1, N}-\lambda_{n, N}\right|+\lambda_{n+1, N}\left\|U_{n+1, N-1} t_{n}-U_{n, N-1} t_{n}\right\|,
\end{aligned}
$$

where $M_{3}$ is an approximate constant such that

$$
M_{3} \geq \max \left\{\sup _{n \geq 1}\left\{\left\|t_{n}\right\|\right\}, \sup _{n \geq 1}\left\{\left\|T_{k} U_{n, k-1} t_{n}\right\|\right\} \mid k=1,2, \ldots, N\right\} .
$$


Since $0<\lambda_{n_{i}} \leq 1$ for all $n \geq 1$ and $i=1,2, \ldots, N$, we have

$$
\begin{aligned}
&\left\|U_{n+1, N-1} t_{n}-U_{n, N-1} t_{n}\right\| \\
&=\left\|\lambda_{n+1, N-1} T_{N-1} U_{n+1, N-2} t_{n}+\left(1-\lambda_{n+1, N-1}\right) t_{n}-\lambda_{n, N-1} T_{N-1} U_{n, N-2} t_{n}-\left(1-\lambda_{n, N-1}\right) t_{n}\right\| \\
& \leq\left|\lambda_{n+1, N-1}-\lambda_{n, N-1}\right|\left\|t_{n}\right\|+\left\|\lambda_{n+1, N-1} T_{N-1} U_{n+1, N-2} t_{n}-\lambda_{n, N-1} T_{N-1} U_{n, N-2} t_{n}\right\| \\
& \leq\left|\lambda_{n+1, N-1}-\lambda_{n, N-1}\right|\left\|t_{n}\right\|+\lambda_{n+1, N-1}\left\|T_{N-1} U_{n+1, N-2} t_{n}-T_{N-1} U_{n, N-2} t_{n}\right\| \\
&+\left|\lambda_{n+1, N-1}-\lambda_{n, N-1}\right|\left\|T_{N-1} U_{n, N-2} t_{n}\right\| \\
& \leq 2 M_{3}\left|\lambda_{n+1, N-1}-\lambda_{n, N-1}\right|+\left\|U_{n+1, N-2} t_{n}-U_{n, N-2} t_{n}\right\| .
\end{aligned}
$$

It follows that

$$
\begin{aligned}
& \left\|U_{n+1, N-1} t_{n}-U_{n, N-1} t_{n}\right\| \\
& \quad \leq 2 M_{3}\left|\lambda_{n+1, N-1}-\lambda_{n, N-1}\right|+2 M_{3}\left|\lambda_{n+1, N-2}-\lambda_{n, N-2}\right|+\left\|U_{n+1, N-3} t_{n}-U_{n, N-3} t_{n}\right\| \\
& \quad \leq 2 M_{3} \sum_{i=2}^{N-1}\left|\lambda_{n+1, i}-\lambda_{n, i}\right|+\left\|U_{n+1,1} t_{n}-U_{n, 1} t_{n}\right\| \\
& \quad=2 M_{3} \sum_{i=2}^{N-1}\left|\lambda_{n+1, i}-\lambda_{n, i}\right|+\left\|\lambda_{n+1,1} T_{1} t_{n}+\left(1-\lambda_{n+1,1}\right) t_{n}-\lambda_{n, 1} T_{1} t_{n}-\left(1-\lambda_{n, 1}\right) t_{n}\right\| \\
& \leq 2 M_{3} \sum_{i=1}^{N-1}\left|\lambda_{n+1, i}-\lambda_{n, i}\right| .
\end{aligned}
$$

Substituting (3.24) into (3.21) yields that

$$
\begin{aligned}
\left\|W_{n+1} t_{n}-W_{n} t_{n}\right\| & \leq 2 M_{3}\left|\lambda_{n+1, N}-\lambda_{n, N}\right|+2 \lambda_{n+1, N} M_{3} \sum_{i=1}^{N-1}\left|\lambda_{n+1, i}-\lambda_{n, i}\right| \\
& \leq 2 M_{3} \sum_{i=1}^{N}\left|\lambda_{n+1, i}-\lambda_{n, i}\right| .
\end{aligned}
$$

Hence, we have

$$
\begin{aligned}
\left\|W_{n+1} t_{n+1}-W_{n} t_{n}\right\| & \leq\left\|W_{n+1} t_{n+1}-W_{n+1} t_{n}\right\|+\left\|W_{n+1} t_{n}-W_{n} t_{n}\right\| \\
& \leq\left\|t_{n+1}-t_{n}\right\|+2 M_{3} \sum_{i=1}^{N}\left|\lambda_{n+1, i}-\lambda_{n, i}\right| .
\end{aligned}
$$


From (3.20), (3.26), and (3.18), we have

$$
\begin{aligned}
\| v_{n+1}- & v_{n}\|-\| x_{n+1}-x_{n} \| \\
\leq & \frac{\alpha_{n+1}}{1-\beta_{n+1}}\left(\left\|f\left(x_{n+1}\right)\right\|+\left\|W_{n+1} t_{n+1}\right\|\right)+\frac{\alpha_{n}}{1-\beta_{n}}\left(\left\|f\left(x_{n}\right)\right\|+\left\|W_{n} t_{n}\right\|\right) \\
& +\left\|W_{n+1} t_{n+1}-W_{n} t_{n}\right\|-\left\|x_{n+1}-x_{n}\right\| \\
\leq & \frac{\alpha_{n+1}}{1-\beta_{n+1}}\left(\left\|f\left(x_{n+1}\right)\right\|+\left\|W_{n+1} t_{n+1}\right\|\right)+\frac{\alpha_{n}}{1-\beta_{n}}\left(\left\|f\left(x_{n}\right)\right\|+\left\|W_{n} t_{n}\right\|\right) \\
& +\left\|t_{n+1}-t_{n}\right\|+2 M_{3} \sum_{i=1}^{N}\left|\lambda_{n+1, i}-\lambda_{n, i}\right|-\left\|x_{n+1}-x_{n}\right\| \\
\leq & \frac{\alpha_{n+1}}{1-\beta_{n+1}}\left(\left\|f\left(x_{n+1}\right)\right\|+\left\|W_{n+1} t_{n+1}\right\|\right)+\frac{\alpha_{n}}{1-\beta_{n}}\left(\left\|f\left(x_{n}\right)\right\|+\left\|W_{n} t_{n}\right\|\right)+2 M_{3} \sum_{i=1}^{N}\left|\lambda_{n+1, i}-\lambda_{n, i}\right| \\
& +\frac{1}{b}\left|r_{n+1}-r_{n}\right| M_{2}+\left(\gamma_{n+1}+\gamma_{n}\right) M_{1} .
\end{aligned}
$$

It follows from (C1)-(C5) that

$$
\limsup _{n \rightarrow \infty}\left(\left\|v_{n+1}-v_{n}\right\|-\left\|x_{n+1}-x_{n}\right\|\right) \leq 0 .
$$

Hence by Lemma 2.4, we have $\lim _{n \rightarrow \infty}\left\|v_{n}-x_{n}\right\|=0$. Consequently

$$
\lim _{n \rightarrow \infty}\left\|x_{n+1}-x_{n}\right\|=\lim _{n \rightarrow \infty}\left(1-\beta_{n}\right)\left\|v_{n}-x_{n}\right\|=0 .
$$

Since $x_{n+1}=\alpha_{n} f\left(x_{n}\right)+\beta_{n} x_{n}+\left(1-\alpha_{n}-\beta_{n}\right) W_{n} t_{n}$, we have

$$
\begin{aligned}
\left\|x_{n}-W_{n} t_{n}\right\| & \leq\left\|x_{n+1}-x_{n}\right\|+\left\|x_{n+1}-W_{n} t_{n}\right\| \\
& \leq\left\|x_{n+1}-x_{n}\right\|+\alpha_{n}\left\|f\left(x_{n}\right)-W_{n} t_{n}\right\|+\beta_{n}\left\|x_{n}-W_{n} t_{n}\right\|,
\end{aligned}
$$

and thus

$$
\left\|x_{n}-W_{n} t_{n}\right\| \leq \frac{1}{1-\beta_{n}}\left(\left\|x_{n+1}-x_{n}\right\|+\alpha_{n}\left\|f\left(x_{n}\right)-W_{n} t_{n}\right\|\right) .
$$

It follows from (C1) and (C2) that $\lim _{n \rightarrow \infty}\left\|x_{n}-W_{n} t_{n}\right\|=0$. 
Fixed Point Theory and Applications

Since $x_{n+1}=\alpha_{n} f\left(x_{n}\right)+\beta_{n} x_{n}+\left(1-\alpha_{n}-\beta_{n}\right) W_{n} t_{n}$, for $u \in \Omega$, it follows from (3.3) and (3.6) that

$$
\begin{aligned}
\left\|x_{n+1}-u\right\|^{2} & =\left\|\alpha_{n} f\left(x_{n}\right)+\beta_{n} x_{n}+\left(1-\alpha_{n}-\beta_{n}\right) W_{n} t_{n}-u\right\|^{2} \\
& \leq \alpha_{n}\left\|f\left(x_{n}\right)-u\right\|^{2}+\beta_{n}\left\|x_{n}-u\right\|^{2}+\left(1-\alpha_{n}-\beta_{n}\right)\left\|W_{n} t_{n}-u\right\|^{2} \\
& \leq \alpha_{n}\left\|f\left(x_{n}\right)-u\right\|^{2}+\beta_{n}\left\|x_{n}-u\right\|^{2}+\left(1-\alpha_{n}-\beta_{n}\right)\left\|t_{n}-u\right\|^{2} \\
& \leq \alpha_{n}\left\|f\left(x_{n}\right)-u\right\|^{2}+\beta_{n}\left\|x_{n}-u\right\|^{2}+\left(1-\alpha_{n}-\beta_{n}\right)\left[\left\|u_{n}-u\right\|^{2}+\left(r_{n}{ }^{2} k^{2}-1\right)\left\|u_{n}-y_{n}\right\|^{2}\right] \\
& \leq \alpha_{n}\left\|f\left(x_{n}\right)-u\right\|^{2}+\left(1-\alpha_{n}\right)\left\|x_{n}-u\right\|^{2}+\left(1-\alpha_{n}-\beta_{n}\right)\left(r_{n}{ }^{2} k^{2}-1\right)\left\|u_{n}-y_{n}\right\|^{2}
\end{aligned}
$$

from which it follows that

$$
\begin{aligned}
\left\|u_{n}-y_{n}\right\|^{2} \leq & \frac{\alpha_{n}}{\left(1-\alpha_{n}-\beta_{n}\right)\left(1-\gamma_{n}{ }^{2} k^{2}\right)}\left(\left\|f\left(x_{n}\right)-u\right\|^{2}-\left\|x_{n}-u\right\|^{2}\right) \\
& +\frac{1}{\left(1-\alpha_{n}-\beta_{n}\right)\left(1-\gamma_{n}{ }^{2} k^{2}\right)}\left(\left\|x_{n}-u\right\|^{2}-\left\|x_{n+1}-u\right\|^{2}\right) \\
\leq & \frac{\alpha_{n}}{\left(1-\alpha_{n}-\beta_{n}\right)\left(1-\gamma_{n}{ }^{2} k^{2}\right)}\left(\left\|f\left(x_{n}\right)-u\right\|^{2}-\left\|x_{n}-u\right\|^{2}\right) \\
& +\frac{1}{\left(1-\alpha_{n}-\beta_{n}\right)\left(1-\gamma_{n}{ }^{2} k^{2}\right)}\left(\left\|x_{n}-u\right\|-\left\|x_{n+1}-u\right\|\right)\left\|x_{n+1}-x_{n}\right\| .
\end{aligned}
$$

It follows from (C1)-(C3) and $\left\|x_{n+1}-x_{n}\right\| \rightarrow 0$ that $\left\|u_{n}-y_{n}\right\| \rightarrow 0$.

By the same argument as in (3.6), we also have

$$
\begin{aligned}
\left\|t_{n}-u\right\|^{2} & \leq\left\|u_{n}-u\right\|^{2}-\left\|u_{n}-y_{n}\right\|^{2}-\left\|y_{n}-t_{n}\right\|^{2}+2 \gamma_{n} k\left\|u_{n}-y_{n}\right\|\left\|t_{n}-y_{n}\right\| \\
& \leq\left\|u_{n}-u\right\|^{2}-\left\|u_{n}-y_{n}\right\|^{2}-\left\|y_{n}-t_{n}\right\|^{2}+\left\|u_{n}-y_{n}\right\|^{2}+\gamma_{n}{ }^{2} k^{2}\left\|t_{n}-y_{n}\right\|^{2} \\
& =\left\|u_{n}-u\right\|^{2}+\left(\gamma_{n}{ }^{2} k^{2}-1\right)\left\|y_{n}-t_{n}\right\|^{2} .
\end{aligned}
$$

Combining the above inequality and (3.32), we have

$$
\begin{aligned}
\left\|x_{n+1}-u\right\|^{2} & \leq \alpha_{n}\left\|f\left(x_{n}\right)-u\right\|^{2}+\beta_{n}\left\|x_{n}-u\right\|^{2}+\left(1-\alpha_{n}-\beta_{n}\right)\left\|t_{n}-u\right\|^{2} \\
& \leq \alpha_{n}\left\|f\left(x_{n}\right)-u\right\|^{2}+\beta_{n}\left\|x_{n}-u\right\|^{2}+\left(1-\alpha_{n}-\beta_{n}\right)\left[\left\|u_{n}-u\right\|^{2}+\left(\gamma_{n}{ }^{2} k^{2}-1\right)\left\|y_{n}-t_{n}\right\|^{2}\right] \\
& \leq \alpha_{n}\left\|f\left(x_{n}\right)-u\right\|^{2}+\left(1-\alpha_{n}\right)\left\|x_{n}-u\right\|^{2}+\left(1-\alpha_{n}-\beta_{n}\right)\left(\gamma_{n}{ }^{2} k^{2}-1\right)\left\|y_{n}-t_{n}\right\|^{2},
\end{aligned}
$$


and thus

$$
\begin{aligned}
\left\|t_{n}-y_{n}\right\|^{2} \leq & \frac{\alpha_{n}}{\left(1-\alpha_{n}-\beta_{n}\right)\left(1-\gamma_{n}{ }^{2} k^{2}\right)}\left(\left\|f\left(x_{n}\right)-u\right\|^{2}-\left\|x_{n}-u\right\|^{2}\right) \\
& +\frac{1}{\left(1-\alpha_{n}-\beta_{n}\right)\left(1-\gamma_{n}{ }^{2} k^{2}\right)}\left(\left\|x_{n}-u\right\|^{2}-\left\|x_{n+1}-u\right\|^{2}\right) \\
\leq & \frac{\alpha_{n}}{\left(1-\alpha_{n}-\beta_{n}\right)\left(1-\gamma_{n}{ }^{2} k^{2}\right)}\left(\left\|f\left(x_{n}\right)-u\right\|^{2}-\left\|x_{n}-u\right\|^{2}\right) \\
& +\frac{1}{\left(1-\alpha_{n}-\beta_{n}\right)\left(1-\gamma_{n}{ }^{2} k^{2}\right)}\left(\left\|x_{n}-u\right\|-\left\|x_{n+1}-u\right\|\right)\left\|x_{n+1}-x_{n}\right\|,
\end{aligned}
$$

which implies that $\left\|t_{n}-y_{n}\right\| \rightarrow 0$.

From $\left\|u_{n}-t_{n}\right\| \leq\left\|u_{n}-y_{n}\right\|+\left\|y_{n}-t_{n}\right\|$ we also have $\left\|u_{n}-t_{n}\right\| \rightarrow 0$. As $A$ is $k$-Lipschitz continuous, we have $\left\|A y_{n}-A t_{n}\right\| \rightarrow 0$.

For $u \in \Omega$, we have, from Lemma 2.1,

$$
\begin{aligned}
\left\|u_{n}-u\right\|^{2} & =\left\|T_{r_{n}} x_{n}-T_{r_{n}} u\right\|^{2} \leq\left\langle T_{r_{n}} x_{n}-T_{r_{n}} u, x_{n}-u\right\rangle \\
& =\left\langle u_{n}-u, x_{n}-u\right\rangle=\frac{1}{2}\left\{\left\|u_{n}-u\right\|^{2}+\left\|x_{n}-u\right\|^{2}-\left\|x_{n}-u_{n}\right\|^{2}\right\} .
\end{aligned}
$$

Hence,

$$
\left\|u_{n}-u\right\|^{2} \leq\left\|x_{n}-u\right\|^{2}-\left\|x_{n}-u_{n}\right\|^{2}
$$

By(3.3), (3.6), (3.32), and (3.38), we have

$$
\begin{aligned}
\left\|x_{n+1}-u\right\|^{2} & \leq \alpha_{n}\left\|f\left(x_{n}\right)-u\right\|^{2}+\beta_{n}\left\|x_{n}-u\right\|^{2}+\left(1-\alpha_{n}-\beta_{n}\right)\left\|t_{n}-u\right\|^{2} \\
& \leq \alpha_{n}\left\|f\left(x_{n}\right)-u\right\|^{2}+\beta_{n}\left\|x_{n}-u\right\|^{2}+\left(1-\alpha_{n}-\beta_{n}\right)\left\|u_{n}-u\right\|^{2} \\
& \leq \alpha_{n}\left\|f\left(x_{n}\right)-u\right\|^{2}+\beta_{n}\left\|x_{n}-u\right\|^{2}+\left(1-\alpha_{n}-\beta_{n}\right)\left[\left\|x_{n}-u\right\|^{2}-\left\|x_{n}-u_{n}\right\|^{2}\right] \\
& \leq \alpha_{n}\left\|f\left(x_{n}\right)-u\right\|^{2}+\left(1-\alpha_{n}\right)\left\|x_{n}-u\right\|^{2}-\left(1-\alpha_{n}-\beta_{n}\right)\left\|x_{n}-u_{n}\right\|^{2} .
\end{aligned}
$$

Hence,

$$
\begin{aligned}
\left(1-\alpha_{n}-\beta_{n}\right)\left\|x_{n}-u_{n}\right\|^{2} & \leq \alpha_{n}\left\|f\left(x_{n}\right)-u\right\|^{2}-\alpha_{n}\left\|x_{n}-u\right\|^{2}+\left\|x_{n}-u\right\|^{2}-\left\|x_{n+1}-u\right\|^{2} \\
& \leq \alpha_{n}\left\|f\left(x_{n}\right)-u\right\|^{2}-\alpha_{n}\left\|x_{n}-u\right\|^{2}+\left(\left\|x_{n}-u\right\|+\left\|x_{n+1}-u\right\|\right)\left\|x_{n}-x_{n+1}\right\| .
\end{aligned}
$$

It follows from (C1), (C2), and $\left\|x_{n}-x_{n+1}\right\| \rightarrow 0$ that $\lim _{n \rightarrow \infty}\left\|x_{n}-u_{n}\right\|=0$. 
Fixed Point Theory and Applications

Since

$$
\begin{aligned}
\left\|W_{n} y_{n}-y_{n}\right\| & \leq\left\|W_{n} y_{n}-W_{n} t_{n}\right\|+\left\|W_{n} t_{n}-x_{n}\right\|+\left\|x_{n}-u_{n}\right\|+\left\|u_{n}-y_{n}\right\| \\
& \leq\left\|y_{n}-t_{n}\right\|+\left\|W_{n} t_{n}-x_{n}\right\|+\left\|x_{n}-u_{n}\right\|+\left\|u_{n}-y_{n}\right\| .
\end{aligned}
$$

It follows that

$$
\lim _{n \rightarrow \infty}\left\|W_{n} y_{n}-y_{n}\right\|=0
$$

Next we show that

$$
\limsup _{n \rightarrow \infty}\left\langle f\left(u_{0}\right)-u_{0}, x_{n}-u_{0}\right\rangle \leq 0
$$

where $u_{0}=P_{\Omega} f\left(u_{0}\right)$. To show this inequality, we can choose a subsequence $\left\{x_{n_{j}}\right\}$ of $\left\{x_{n}\right\}$ such that

$$
\lim _{n \rightarrow \infty}\left\langle f\left(u_{0}\right)-u_{0}, x_{n_{j}}-u_{0}\right\rangle=\limsup _{n \rightarrow \infty}\left\langle f\left(u_{0}\right)-u_{0}, x_{n}-u_{0}\right\rangle .
$$

Since $\left\{x_{n i}\right\}$ is bounded, there exists a subsequence $\left\{x_{n i j}\right\}$ of $\left\{x_{n i}\right\}$ which converges weakly to $w$. Without loss of generality, we can assume that $\left\{x_{n i}\right\} \rightarrow w$. From $\left\|x_{n}-u_{n}\right\| \rightarrow 0$, we obtain that $u_{n i} \rightarrow w$. From $\left\|u_{n}-y_{n}\right\| \rightarrow 0$, we also obtain that $y_{n_{i}} \rightarrow w$. From $\left\|u_{n}-t_{n}\right\| \rightarrow 0$, we also obtain that $t_{n i} \rightarrow w$. Since $\left\{u_{n i}\right\} \subset C$ and $C$ is closed and convex, we obtain $w \in C$.

In order to show that $w \in \Omega$, we first show $w \in \operatorname{MEP}(F, \varphi)$. By $u_{n}=T_{r_{n}} x_{n}$, we know that

$$
F\left(u_{n}, y\right)+\varphi(y)-\varphi\left(u_{n}\right)+\frac{1}{r_{n}}\left\langle y-u_{n}, u_{n}-x_{n}\right\rangle \geq 0, \quad \forall y \in C
$$

It follows from (A2) that

$$
\varphi(y)-\varphi\left(u_{n}\right)+\frac{1}{r_{n}}\left\langle y-u_{n}, u_{n}-x_{n}\right\rangle \geq F\left(y, u_{n}\right), \quad \forall y \in C
$$

Hence,

$$
\varphi(y)-\varphi\left(u_{n_{i}}\right)+\left\langle y-u_{n_{i}}, \frac{u_{n_{i}}-x_{n_{i}}}{r_{n_{i}}}\right\rangle \geq F\left(y, u_{n_{i}}\right), \quad \forall y \in C
$$

It follows from (A4), (A5), and the weakly lower semicontinuity of $\varphi,\left(u_{n_{i}}-x_{n_{i}}\right) / r_{n_{i}} \rightarrow$ 0 and $u_{n_{i}} \rightarrow w$ that

$$
F(y, w)+\varphi(w)-\varphi(y) \leq 0, \quad \forall y \in C
$$


For $t$ with $0<t \leq 1$ and $y \in C$, let $y_{t}=t y+(1-t) w$. Since $y \in C$ and $w \in C$, we obtain $y_{t} \in C$ and hence $F\left(y_{t}, w\right)+\varphi(w)-\varphi\left(y_{t}\right) \leq 0$. So by (A4) and the convexity of $\varphi$, we have

$$
\begin{aligned}
0 & =F\left(y_{t}, y_{t}\right)+\varphi\left(y_{t}\right)-\varphi\left(y_{t}\right) \\
& \leq t F\left(y_{t}, y\right)+(1-t) F\left(y_{t}, w\right)+t \varphi(y)+(1-t) \varphi(w)-\varphi\left(y_{t}\right) \\
& \leq t\left[F\left(y_{t}, y\right)+\varphi(y)-\varphi\left(y_{t}\right)\right] .
\end{aligned}
$$

Dividing by $t$, we get

$$
F\left(y_{t}, y\right)+\varphi(y)-\varphi\left(y_{t}\right) \geq 0
$$

Letting $t \rightarrow 0$, it follows from (A3) and the weakly lower semicontinuity of $\varphi$ that

$$
F(w, y)+\varphi(y)-\varphi(w) \geq 0
$$

for all $y \in C$ and hence $w \in \operatorname{MEP}(F, \varphi)$.

Now we show that $w \in \operatorname{VI}(C, A)$. Put

$$
T w_{1}= \begin{cases}A w_{1}+N_{C} w_{1} & \text { if } w_{1} \in C, \\ \emptyset & \text { if } w_{1} \notin C\end{cases}
$$

where $N_{C} w_{1}$ is the normal cone to $C$ at $w_{1} \in C$. We have already mentioned that in this case the mapping $T$ is maximal monotone, and $0 \in T w_{1}$ if and only if $w_{1} \in \operatorname{VI}(C, A)$. Let $\left(w_{1}, g\right) \in$ $G(T)$. Then $T w_{1}=A w_{1}+N_{C} w_{1}$ and hence $g-A w_{1} \in N_{C} w_{1}$. So, we have $\left\langle w_{1}-t, g-A w_{1}\right\rangle \geq 0$ for all $t \in C$. On the other hand, from $t_{n}=P_{C}\left(u_{n}-\lambda_{n} A y_{n}\right)$ and $w_{1} \in C$ we have

$$
\left\langle u_{n}-\lambda_{n} A y_{n}-t_{n}, t_{n}-w_{1}\right\rangle \geq 0,
$$

and hence

$$
\left\langle w_{1}-t_{n}, \frac{t_{n}-u_{n}}{\lambda_{n}}+A y_{n}\right\rangle \geq 0
$$


Therefore, we have

$$
\begin{aligned}
\left\langle w_{1}-t_{n_{i}}, g\right\rangle & \geq\left\langle w_{1}-t_{n_{i}}, A w_{1}\right\rangle \\
& \geq\left\langle w_{1}-t_{n_{i}}, A w_{1}\right\rangle-\left\langle w_{1}-t_{n_{i}}, \frac{t_{n_{i}}-u_{n_{i}}}{\lambda_{n_{i}}}+A y_{n_{i}}\right\rangle \\
& =\left\langle w_{1}-t_{n_{i}}, A w_{1}-A y_{n_{i}}-\frac{t_{n_{i}}-u_{n_{i}}}{\lambda_{n_{i}}}\right\rangle \\
& =\left\langle w_{1}-t_{n_{i}}, A w_{1}-A t_{n_{i}}+A t_{n_{i}}-A y_{n_{i}}-\frac{t_{n_{i}}-u_{n_{i}}}{\lambda_{n_{i}}}\right\rangle \\
& =\left\langle w_{1}-t_{n_{i}}, A w_{1}-A t_{n_{i}}\right\rangle+\left\langle w_{1}-t_{n_{i}}, A t_{n_{i}}-A y_{n_{i}}\right\rangle-\left\langle v-t_{n_{i}}, \frac{t_{n_{i}}-u_{n_{i}}}{\lambda_{n_{i}}}\right\rangle \\
& \geq\left\langle w_{1}-t_{n_{i}}, A t_{n_{i}}-A y_{n_{i}}\right\rangle-\left\langle w_{1}-t_{n_{i}}, \frac{t_{n_{i}}-u_{n_{i}}}{\lambda_{n_{i}}}\right\rangle .
\end{aligned}
$$

Hence we obtain $\left\langle w_{1}-w, g\right\rangle \geq 0$ as $i \rightarrow \infty$. Since $T$ is maximal monotone, we have $w \in T^{-1} 0$ and hence $w \in \operatorname{VI}(C, A)$.

We next show that $w \in \bigcap_{i=1}^{N} \operatorname{Fix}\left(T_{i}\right)$. To see this, we observe that we may assume (by passing to a further subsequence if necessary) $\lambda_{n_{i}, k} \rightarrow \lambda_{k}$ for $k=1,2, \ldots, N$. Let $W$ be the $W$-mapping generated by $T_{1}, T_{2}, \ldots, T_{N}$ and $\lambda_{1}, \lambda_{2}, \ldots, \lambda_{N}$. By Lemma 2.5 , we know that $W$ is nonexpansive and $\bigcap_{i=1}^{N} \operatorname{Fix}\left(T_{i}\right)=\operatorname{Fix}(W)$. it follows from Lemma 2.6 that

$$
W_{n_{i}} x \longrightarrow W x, \quad \forall x \in C
$$

Assume $w \notin \operatorname{Fix}(W)$. Since $x_{n_{i}} \rightarrow w$ and $w \neq W w$, it follows from the Opial condition, (3.42), and (3.56) that

$$
\begin{aligned}
\liminf _{i \rightarrow \infty}\left\|x_{n_{i}}-w\right\| & <\liminf _{i \rightarrow \infty}\left\|x_{n_{i}}-W w\right\| \\
& \leq \liminf _{i \rightarrow \infty}\left\{\left\|x_{n_{i}}-W_{n_{i}} x_{n_{i}}\right\|+\left\|W_{n_{i}} x_{n_{i}}-W x_{n_{i}}\right\|+\left\|W x_{n_{i}}-W w\right\|\right\} \\
& \leq \liminf _{i \rightarrow \infty}\left\|x_{n_{i}}-w\right\|,
\end{aligned}
$$

which is a contradiction. Hence, we have $w \in \operatorname{Fix}(W)=\bigcap_{i=1}^{N} \operatorname{Fix}\left(T_{i}\right)$. This implies $w \in \Omega$. Therefore, we have

$$
\limsup _{n \rightarrow \infty}\left\langle f\left(u_{0}\right)-u_{0}, x_{n}-u_{0}\right\rangle=\lim _{j \rightarrow \infty}\left\langle f\left(u_{0}\right)-u_{0}, x_{n_{j}}-u_{0}\right\rangle=\left\langle f\left(u_{0}\right)-u_{0}, w-u_{0}\right\rangle \leq 0 .
$$

Finally, we show that $x_{n} \rightarrow u_{0}$, where $u_{0}=P_{\Omega} f\left(u_{0}\right)$. 
From Lemma 2.3, we have

$$
\begin{aligned}
\left\|x_{n+1}-u_{0}\right\|^{2}= & \left\|\alpha_{n}\left(f\left(x_{n}\right)-u_{0}\right)+\beta_{n}\left(x_{n}-u_{0}\right)+\left(1-\alpha_{n}-\beta_{n}\right)\left(W_{n} t_{n}-u_{0}\right)\right\|^{2} \\
\leq & \left\|\beta_{n}\left(x_{n}-u_{0}\right)+\left(1-\alpha_{n}-\beta_{n}\right)\left(W_{n} t_{n}-u_{0}\right)\right\|^{2}+2 \alpha_{n}\left\langle f\left(x_{n}\right)-u_{0}, x_{n+1}-u_{0}\right\rangle \\
\leq & \left(1-\alpha_{n}-\beta_{n}\right)\left\|W_{n} t_{n}-u_{0}\right\|^{2}+\beta_{n}\left\|x_{n}-u_{0}\right\|^{2}+2 \alpha_{n}\left\langle f\left(x_{n}\right)-u_{0}, x_{n+1}-u_{0}\right\rangle \\
\leq & \left(1-\alpha_{n}-\beta_{n}\right)\left\|W_{n} t_{n}-u_{0}\right\|^{2}+\beta_{n}\left\|x_{n}-u_{0}\right\|^{2}+2 \alpha_{n}\left\langle f\left(x_{n}\right)-f\left(u_{0}\right), x_{n+1}-u_{0}\right\rangle \\
& +2 \alpha_{n}\left\langle f\left(u_{0}\right)-u_{0}, x_{n+1}-u_{0}\right\rangle \\
\leq & \left(1-\alpha_{n}-\beta_{n}\right)\left\|t_{n}-u_{0}\right\|^{2}+\beta_{n}\left\|x_{n}-u_{0}\right\|^{2}+2 \alpha_{n} a\left\|x_{n}-u_{0}\right\|\left\|x_{n+1}-u_{0}\right\| \\
& +2 \alpha_{n}\left\langle f\left(u_{0}\right)-u_{0}, x_{n+1}-u_{0}\right\rangle \\
\leq & \left(1-\alpha_{n}\right)\left\|x_{n}-u_{0}\right\|^{2}+\alpha_{n} a\left(\left\|x_{n}-u_{0}\right\|^{2}+\left\|x_{n+1}-u_{0}\right\|^{2}\right)+2 \alpha_{n}\left\langle f\left(u_{0}\right)-u_{0}, x_{n+1}-u_{0}\right\rangle,
\end{aligned}
$$

and thus

$$
\left\|x_{n+1}-u_{0}\right\|^{2} \leq\left(1-\frac{\alpha_{n}}{1-a \alpha_{n}}\right)\left\|x_{n}-u_{0}\right\|^{2}+\frac{\alpha_{n}}{1-a \alpha_{n}}\left\langle 2 f\left(u_{0}\right)-2 u_{0}, x_{n+1}-u_{0}\right\rangle
$$

It follows from Lemma 2.2, (3.58), and (3.60) that $\lim _{n \rightarrow \infty}\left\|x_{n}-u_{0}\right\|=0$. From $\| x_{n}-$ $u_{n} \| \rightarrow 0$ and $\left\|y_{n}-u_{n}\right\| \rightarrow 0$, we have $u_{n} \rightarrow u_{0}$ and $y_{n} \rightarrow u_{0}$. The proof is now complete.

\section{Applications}

By Theorem 3.1, we can obtain some new and interesting strong convergence theorems as follows.

Theorem 4.1. Let $C$ be a nonempty closed convex subset of a real Hilbert space $H$. Let $F$ be a bifunction from $C \times C$ to $R$ satisfying (A1)-(A5) and let $\varphi: C \rightarrow R \cup\{+\infty\}$ be a proper lower semicontinuous and convex function. Let $T_{1}, T_{2}, \ldots, T_{N}$ be a finite family of nonexpansive mappings of $C$ into $H$ such that $\Sigma=\bigcap_{n=1}^{N} \operatorname{Fix}\left(T_{i}\right) \cap \operatorname{MEP}(F, \varphi) \neq \emptyset$. Let $\left\{\lambda_{n, 1}\right\},\left\{\lambda_{n, 2}\right\}, \ldots,\left\{\lambda_{n, N}\right\}$ be sequences in $\left[\varepsilon_{1}, \varepsilon_{2}\right]$ with $0<\varepsilon_{1} \leq \varepsilon_{2}<1$. Let $W_{n}$ be the $W$-mapping generated by $T_{1}, T_{2}, \ldots, T_{N}$ and $\lambda_{n, 1}, \lambda_{n, 2}, \ldots, \lambda_{n, N}$. Assume that either (B1) or (B2) holds. Let $f$ be a contraction of $H$ into itself and let $\left\{x_{n}\right\},\left\{u_{n}\right\}$, and $\left\{y_{n}\right\}$ be sequences generated by

$$
\begin{gathered}
x_{1}=x \in C, \\
F\left(u_{n}, y\right)+\varphi(y)-\varphi\left(u_{n}\right)+\frac{1}{r_{n}}\left\langle y-u_{n}, u_{n}-x_{n}\right\rangle \geq 0, \quad \forall y \in C \\
x_{n+1}=\alpha_{n} f\left(x_{n}\right)+\beta_{n} x_{n}+\left(1-\alpha_{n}-\beta_{n}\right) W_{n} u_{n}
\end{gathered}
$$


for every $n=1,2, \ldots$ where $\left\{r_{n}\right\},\left\{\alpha_{n}\right\},\left\{\lambda_{n 1}\right\},\left\{\lambda_{n 2}\right\}, \ldots,\left\{\lambda_{n N}\right\}$, and $\left\{\beta_{n}\right\}$ are sequences of numbers satisfying the following conditions:

(C1) $\lim _{n \rightarrow \infty} \alpha_{n}=0$ and $\sum_{n=1}^{\infty} \alpha_{n}=\infty$;

(C2) $1>\lim \sup _{n \rightarrow \infty} \beta_{n} \geq \liminf _{n \rightarrow \infty} \beta_{n}>0$;

(C4) $\liminf _{n \rightarrow \infty} r_{n}>0$ and $\lim _{n \rightarrow \infty}\left|r_{n+1}-r_{n}\right|=0$;

(C5) $\lim _{n \rightarrow \infty}\left|\lambda_{n, i}-\lambda_{n-1, i}\right|=0$ for all $i=1,2, \ldots, N$.

Then, $\left\{x_{n}\right\},\left\{u_{n}\right\}$, and $\left\{y_{n}\right\}$ converge strongly to $w=P_{\Sigma} f(w)$.

Proof. Putting $A=0$, by Theorem 3.1 we obtain the desired result.

Theorem 4.2. Let $C$ be a nonempty closed convex subset of a real Hilbert space $H$. Let $F$ be a bifunction from $C \times C$ to $R$ satisfying (A1)-(A5). Let $A$ be a monotone and $k$-Lipschitz continuous mapping of $C$ into $H$. Let $T_{1}, T_{2}, \ldots, T_{N}$ be a finite family of nonexpansive mappings of $C$ into $H$ such that $\Lambda=\bigcap_{n=1}^{N} \operatorname{Fix}\left(T_{i}\right) \cap \operatorname{VI}(C, A) \cap E P(F) \neq \emptyset$. Let $\left\{\lambda_{n, 1}\right\},\left\{\lambda_{n, 2}\right\}, \ldots,\left\{\lambda_{n, N}\right\}$ be sequences in $\left[\varepsilon_{1}, \varepsilon_{2}\right]$ with $0<\varepsilon_{1} \leq \varepsilon_{2}<1$. Let $W_{n}$ be the $W$-mapping generated by $T_{1}, T_{2}, \ldots, T_{N}$ and $\lambda_{n, 1}, \lambda_{n, 2}, \ldots, \lambda_{n, N}$. Assume that either (B4) or (B2) holds. Let $f$ be a contraction of $H$ into itself and let $\left\{x_{n}\right\},\left\{u_{n}\right\}$, and $\left\{y_{n}\right\}$ be sequences generated by

$$
\begin{gathered}
x_{1}=x \in C, \\
F\left(u_{n}, y\right)+\frac{1}{r_{n}}\left\langle y-u_{n}, u_{n}-x_{n}\right\rangle \geq 0, \quad \forall y \in C, \\
y_{n}=P_{C}\left(u_{n}-\gamma_{n} A u_{n}\right), \\
x_{n+1}=\alpha_{n} f\left(x_{n}\right)+\beta_{n} x_{n}+\left(1-\alpha_{n}-\beta_{n}\right) W_{n} P_{C}\left(u_{n}-\gamma_{n} A y_{n}\right)
\end{gathered}
$$

for every $n=1,2, \ldots$ where $\left\{\gamma_{n}\right\},\left\{r_{n}\right\},\left\{\alpha_{n}\right\},\left\{\lambda_{n 1}\right\},\left\{\lambda_{n 2}\right\}, \ldots,\left\{\lambda_{n N}\right\}$, and $\left\{\beta_{n}\right\}$ are sequences of numbers satisfying the following conditions:

(C1) $\lim _{n \rightarrow \infty} \alpha_{n}=0$ and $\sum_{n=1}^{\infty} \alpha_{n}=\infty$;

(C2) $1>\lim \sup _{n \rightarrow \infty} \beta_{n} \geq \liminf _{n \rightarrow \infty} \beta_{n}>0$;

(C3) $\lim _{n \rightarrow \infty} \gamma_{n}=0$;

(C4) $\liminf _{n \rightarrow \infty} r_{n}>0$ and $\lim _{n \rightarrow \infty}\left|r_{n+1}-r_{n}\right|=0$;

(C5) $\lim _{n \rightarrow \infty}\left|\lambda_{n, i}-\lambda_{n-1, i}\right|=0$ for all $i=1,2, \ldots, N$.

Then, $\left\{x_{n}\right\},\left\{u_{n}\right\}$, and $\left\{y_{n}\right\}$ converge strongly to $w=P_{\Lambda} f(w)$.

Proof. Putting $\varphi=0$, by Theorem 3.1 we obtain the desired result.

Theorem 4.3. Let $C$ be a nonempty closed convex subset of a real Hilbert space $H$. Let $\varphi: C \rightarrow$ $R \cup\{+\infty\}$ be a proper lower semicontinuous and convex function. Let $A$ be a monotone and $k$-Lipschitz continuous mapping of $C$ into $H$. Let $T_{1}, T_{2}, \ldots, T_{N}$ be a finite family of nonexpansive mappings of $C$ into $H$ such that $\Theta=\bigcap_{n=1}^{N} \operatorname{Fix}\left(T_{i}\right) \cap \operatorname{VI}(C, A) \cap \operatorname{Argmin}(\varphi) \neq \emptyset$. Let $\left\{\lambda_{n, 1}\right\},\left\{\lambda_{n, 2}\right\}, \ldots,\left\{\lambda_{n, N}\right\}$ be sequences in $\left[\varepsilon_{1}, \varepsilon_{2}\right]$ with $0<\varepsilon_{1} \leq \varepsilon_{2}<1$. Let $W_{n}$ be the $W$-mapping generated by $T_{1}, T_{2}, \ldots, T_{N}$ and 
$\lambda_{n, 1}, \lambda_{n, 2}, \ldots, \lambda_{n, N}$. Assume that either (B3) or (B2) holds. Let $f$ be a contraction of $H$ into itself and let $\left\{x_{n}\right\},\left\{u_{n}\right\}$, and $\left\{y_{n}\right\}$ be sequences generated by

$$
\begin{gathered}
x_{1}=x \in C, \\
\varphi(y)-\varphi\left(u_{n}\right)+\frac{1}{r_{n}}\left\langle y-u_{n}, u_{n}-x_{n}\right\rangle \geq 0, \quad \forall y \in C, \\
y_{n}=P_{C}\left(u_{n}-\gamma_{n} A u_{n}\right), \\
x_{n+1}=\alpha_{n} f\left(x_{n}\right)+\beta_{n} x_{n}+\left(1-\alpha_{n}-\beta_{n}\right) W_{n} P_{C}\left(u_{n}-\gamma_{n} A y_{n}\right)
\end{gathered}
$$

for every $n=1,2, \ldots$ where $\left\{\gamma_{n}\right\},\left\{r_{n}\right\},\left\{\alpha_{n}\right\},\left\{\lambda_{n 1}\right\},\left\{\lambda_{n 2}\right\}, \ldots,\left\{\lambda_{n N}\right\}$, and $\left\{\beta_{n}\right\}$ are sequences of numbers satisfying the following conditions:

(C1) $\lim _{n \rightarrow \infty} \alpha_{n}=0$ and $\sum_{n=1}^{\infty} \alpha_{n}=\infty$;

(C2) $1>\lim \sup _{n \rightarrow \infty} \beta_{n} \geq \liminf _{n \rightarrow \infty} \beta_{n}>0$;

(C3) $\lim _{n \rightarrow \infty} \gamma_{n}=0$;

(C4) $\liminf _{n \rightarrow \infty} r_{n}>0$ and $\lim _{n \rightarrow \infty}\left|r_{n+1}-r_{n}\right|=0$;

(C5) $\lim _{n \rightarrow \infty}\left|\lambda_{n, i}-\lambda_{n-1, i}\right|=0$ for all $i=1,2, \ldots, N$.

Then, $\left\{x_{n}\right\},\left\{u_{n}\right\}$, and $\left\{y_{n}\right\}$ converge strongly to $w=P_{\Theta} f(w)$.

Proof. Let $F(x, y)=0$ for all $x, y \in C$, by Theorem 3.1 we obtain the desired result.

Theorem 4.4. Let $C$ be a nonempty closed convex subset of a real Hilbert space $H$. Let $F$ be a bifunction from $C \times C$ to $R$ satisfying (A1)-(A5) and let $\varphi: C \rightarrow R \cup\{+\infty\}$ be a proper lower semicontinuous and convex function. Let $A$ be a monotone and $k$-Lipschitz continuous mapping of $C$ into $H$. Let $S$ be a nonexpansive mapping of $C$ into $H$ such that $\operatorname{Fix}(S) \cap \operatorname{VI}(C, A) \cap \operatorname{MEP}(F, \varphi) \neq \emptyset$. Assume that either (B1) or (B2) holds. Let $f$ be a contraction of $H$ into itself and let $\left\{x_{n}\right\},\left\{u_{n}\right\}$, and $\left\{y_{n}\right\}$ be sequences generated by

$$
\begin{gathered}
x_{1}=x \in C, \\
F\left(u_{n}, y\right)+\varphi(y)-\varphi\left(u_{n}\right)+\frac{1}{r_{n}}\left\langle y-u_{n}, u_{n}-x_{n}\right\rangle \geq 0, \quad \forall y \in C, \\
y_{n}=P_{C}\left(u_{n}-\gamma_{n} A u_{n}\right), \\
x_{n+1}=\alpha_{n} f\left(x_{n}\right)+\beta_{n} x_{n}+\left(1-\alpha_{n}-\beta_{n}\right) S P_{C}\left(u_{n}-\gamma_{n} A y_{n}\right)
\end{gathered}
$$

for every $n=1,2, \ldots$ where $\left\{\gamma_{n}\right\},\left\{r_{n}\right\},\left\{\alpha_{n}\right\}$ and $\left\{\beta_{n}\right\}$ are sequences of numbers satisfying the following conditions:

(C1) $\lim _{n \rightarrow \infty} \alpha_{n}=0$ and $\sum_{n=1}^{\infty} \alpha_{n}=\infty$;

(C2) $1>\lim \sup _{n \rightarrow \infty} \beta_{n} \geq \liminf _{n \rightarrow \infty} \beta_{n}>0$;

(C3) $\lim _{n \rightarrow \infty} \gamma_{n}=0$;

(C4) $\liminf _{n \rightarrow \infty} r_{n}>0$ and $\lim _{n \rightarrow \infty}\left|r_{n+1}-r_{n}\right|=0$.

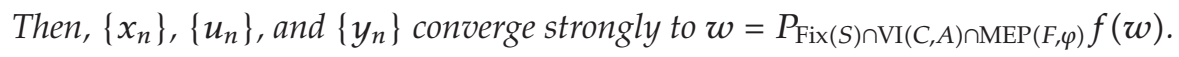

Proof. Let $W_{n}=S$, by Theorem 3.1 we obtain the desired result. 
Theorem 4.5. Let $C$ be a nonempty closed convex subset of a real Hilbert space $H$. Let $A$ be a monotone and $k$-Lipschitz continuous mapping of $C$ into $H$. Let $T_{1}, T_{2}, \ldots, T_{N}$ be a finite family of nonexpansive mappings of $C$ into $H$ such that $\Gamma=\bigcap_{n=1}^{N} \operatorname{Fix}\left(T_{i}\right) \cap \operatorname{VI}(C, A) \neq \emptyset$. Let $\left\{\lambda_{n, 1}\right\},\left\{\lambda_{n, 2}\right\}, \ldots,\left\{\lambda_{n, N}\right\}$ be sequences in $\left[\varepsilon_{1}, \varepsilon_{2}\right]$ with $0<\varepsilon_{1} \leq \varepsilon_{2}<1$. Let $W_{n}$ be the $W$-mapping generated by $T_{1}, T_{2}, \ldots, T_{N}$ and $\lambda_{n, 1}, \lambda_{n, 2}, \ldots, \lambda_{n, N}$. Let $\left\{x_{n}\right\}$ and $\left\{y_{n}\right\}$ be sequences generated by

$$
\begin{gathered}
x_{1}=x \in C, \\
y_{n}=P_{C}\left(x_{n}-\gamma_{n} A x_{n}\right), \\
x_{n+1}=\alpha_{n} f\left(x_{n}\right)+\beta_{n} x_{n}+\left(1-\alpha_{n}-\beta_{n}\right) W_{n} P_{C}\left(x_{n}-\gamma_{n} A y_{n}\right)
\end{gathered}
$$

for every $n=1,2, \ldots$ where $\left\{\gamma_{n}\right\},\left\{\alpha_{n}\right\},\left\{\lambda_{n 1}\right\},\left\{\lambda_{n 2}\right\}, \ldots,\left\{\lambda_{n N}\right\}$, and $\left\{\beta_{n}\right\}$ are sequences of numbers satisfying the following conditions:

(C1) $\lim _{n \rightarrow \infty} \alpha_{n}=0$ and $\sum_{n=1}^{\infty} \alpha_{n}=\infty$;

(C2) $1>\lim \sup _{n \rightarrow \infty} \beta_{n} \geq \liminf _{n \rightarrow \infty} \beta_{n}>0$;

(C3) $\lim _{n \rightarrow \infty} \gamma_{n}=0$;

(C5) $\lim _{n \rightarrow \infty}\left|\lambda_{n, i}-\lambda_{n-1, i}\right|=0$ for all $i=1,2, \ldots, N$.

Then, $\left\{x_{n}\right\}$ and $\left\{y_{n}\right\}$ converge strongly to $w=P_{\Gamma} f(w)$.

Proof. Let $\varphi=0$ and let $F(x, y)=0$ for all $x, y \in C$. Then $u_{n}=P_{C} x_{n}=x_{n}$. By Theorem 3.1 we obtain the desired result.

Remark 4.6. (1) Since the $\alpha$-inverse-strongly-monotonicity of $A$ has been weakened by the monotonicity and Lipschitz continuity of $A$. Theorems 3.1, 4.2, and 4.4 generalize and improve Theorem 3.1 in [11], Theorem 3.1 in [12], and Theorem 3.1 in [8] and the main results in [31]. Theorem 4.5 improves Theorem 3.1 in [20].

(2) It is easy to see that Theorems 3.1, 4.2, and 4.4 also generalize and improve Theorems 3.1, and 4.2 in [9].

(3) It is clear that Theorem 4.5 generalizes, extends, and improves Theorem 3.1 in [18] and Theorem 3.1 in [19].

(4) Theorem 3.1 improves and extends Theorem 3.1 in [1].

\section{Acknowledgments}

The authors would like to express their thanks to the referee for helpful suggestions. This research was supported by the National Center of Theoretical Sciences (South) of Taiwan, the National Natural Science Foundation of China (Grants 10771228 and 10831009), the Natural Science Foundation of Chongqing (Grant no. CSTC, 2009BB8240), and the Research Project of Chongqing Normal University (Grant no. 08XLZ05).

\section{References}

[1] L.-C. Ceng and J.-C. Yao, "A hybrid iterative scheme for mixed equilibrium problems and fixed point problems," Journal of Computational and Applied Mathematics, vol. 214, no. 1, pp. 186-201, 2008.

[2] G. Bigi, M. Castellani, and G. Kassay, "A dual view of equilibrium problems," Journal of Mathematical Analysis and Applications, vol. 342, no. 1, pp. 17-26, 2008. 
[3] S. D. Flam and A. S. Antipin, "Equilibrium programming using proximal-like algorithms," Mathematical Programming, vol. 78, no. 1, pp. 29-41, 1997.

[4] E. Blum and W. Oettli, "From optimization and variational inequalities to equilibrium problems," The Mathematics Student, vol. 63, no. 1-4, pp. 123-145, 1994.

[5] K. Goebel and W. A. Kirk, Topics in Metric Fixed Point Theory, vol. 28 of Cambridge Studies in Advanced Mathematics, Cambridge University Press, Cambridge, UK, 1990.

[6] P. L. Combettes and S. A. Hirstoaga, "Equilibrium programming in Hilbert spaces," Journal of Nonlinear and Convex Analysis, vol. 6, no. 1, pp. 117-136, 2005.

[7] S. Takahashi and W. Takahashi, "Viscosity approximation methods for equilibrium problems and fixed point problems in Hilbert spaces," Journal of Mathematical Analysis and Applications, vol. 331, no. 1, pp. 506-515, 2007.

[8] Y. Su, M. Shang, and X. Qin, "An iterative method of solution for equilibrium and optimization problems," Nonlinear Analysis: Theory, Methods \& Applications, vol. 69, no. 8, pp. 2709-2719, 2008.

[9] A. Tada and W. Takahashi, "Weak and strong convergence theorems for a nonexpansive mapping and an equilibrium problem," Journal of Optimization Theory and Applications, vol. 133, no. 3, pp. 359-370, 2007.

[10] V. Colao, G. Marino, and H.-K. Xu, "An iterative method for finding common solutions of equilibrium and fixed point problems," Journal of Mathematical Analysis and Applications, vol. 344, no. 1, pp. 340 $352,2008$.

[11] S. Plubtieng and R. Punpaeng, "A new iterative method for equilibrium problems and fixed point problems of nonexpansive mappings and monotone mappings," Applied Mathematics and Computation, vol. 197, no. 2, pp. 548-558, 2008.

[12] S.-S. Chang, H. W. Joseph Lee, and C. K. Chan, "A new method for solving equilibrium problem fixed point problem and variational inequality problem with application to optimization," Nonlinear Analysis: Theory, Methods \& Applications, vol. 70, no. 9, pp. 3307-3319, 2009.

[13] G. M. Korpelevich, "The extragradient method for finding saddle points and for other problems," Matecon, vol. 12, no. 4, pp. 747-756, 1976.

[14] B.-S. He, Z.-H. Yang, and X.-M. Yuan, "An approximate proximal-extragradient type method for monotone variational inequalities," Journal of Mathematical Analysis and Applications, vol. 300, no. 2, pp. 362-374, 2004.

[15] R. Gárciga Otero and A. Iusem, "Proximal methods with penalization effects in Banach spaces," Numerical Functional Analysis and Optimization, vol. 25, no. 1-2, pp. 69-91, 2004.

[16] M. V. Solodov and B. F. Svaiter, "An inexact hybrid generalized proximal point algorithm and some new results on the theory of Bregman functions," Mathematics of Operations Research, vol. 25, no. 2, pp. 214-230, 2000.

[17] M. V. Solodov, "Convergence rate analysis of iteractive algorithms for solving variational inquality problems," Mathematical Programming, vol. 96, no. 3, pp. 513-528, 2003.

[18] L.-C. Zeng and J.-C. Yao, "Strong convergence theorem by an extragradient method for fixed point problems and variational inequality problems," Taiwanese Journal of Mathematics, vol. 10, no. 5, pp. 1293-1303, 2006.

[19] N. Nadezhkina and W. Takahashi, "Weak convergence theorem by an extragradient method for nonexpansive mappings and monotone mappings," Journal of Optimization Theory and Applications, vol. 128, no. 1, pp. 191-201, 2006.

[20] Y. Yao and J.-C. Yao, “On modified iterative method for nonexpansive mappings and monotone mappings," Applied Mathematics and Computation, vol. 186, no. 2, pp. 1551-1558, 2007.

[21] J.-W. Peng, Y.-C. Liou, and J.-C. Yao, "An iterative algorithm combining viscosity method with parallel method for a generalized equilibrium problem and strict pseudocontractions," Fixed Point Theory and Applications, vol. 2009, Article ID 794178, 21 pages, 2009.

[22] Z. Opial, "Weak convergence of the sequence of successive approximations for nonexpansive mappings," Bulletin of the American Mathematical Society, vol. 73, pp. 591-597, 1967.

[23] R. T. Rockafellar, "On the maximality of sums of nonlinear monotone operators," Transactions of the American Mathematical Society, vol. 149, pp. 75-88, 1970.

[24] J.-W. Peng and J.-C. Yao, “A new hybrid-extragradient method for generalized mixed equilibrium problems, fixed point problems and variational inequality problems," Taiwanese Journal of Mathematics, vol. 12, no. 6, pp. 1401-1432, 2008.

[25] H.-K. Xu, "Iterative algorithms for nonlinear operators," Journal of the London Mathematical Society, vol. 66, no. 1, pp. 240-256, 2002. 
[26] H.-K. Xu, "An iterative approach to quadratic optimization," Journal of Optimization Theory and Applications, vol. 116, no. 3, pp. 659-678, 2003.

[27] W. Takahashi, "Weak and strong convergence theorems for families of nonexpansive mappings and their applications," Annales Universitatis Mariae Curie-Sklodowska, vol. 51, no. 2, pp. 277-292, 1997.

[28] S. Atsushiba and W. Takahashi, "Strong convergence theorems for a finite family of nonexpansive mappings and applications," Indian Journal of Mathematics, vol. 41, no. 3, pp. 435-453, 1999.

[29] W. Takahashi and K. Shimoji, "Convergence theorems for nonexpansive mappings and feasibility problems," Mathematical and Computer Modelling, vol. 32, no. 11-13, pp. 1463-1471, 2000.

[30] L. C. Ceng, P. Cubiotti, and J. C. Yao, "Strong convergence theorems for finitely many nonexpansive mappings and applications," Nonlinear Analysis: Theory, Methods E Applications, vol. 67, no. 5, pp. 1464-1473, 2007.

[31] C. S. Hu and G. Cai, "Viscosity approximation schemes for fixed point problems and equilibrium problems and variational inequality problems," Nonlinear Analysis: Theory, Methods E Applications, vol. 72, no. 3-4, pp. 1792-1808, 2010. 